Research Article

\title{
Tuberculosis and COVID-19: Lessons from the Past Viral Outbreaks and Possible Future Outcomes
}

\author{
Radu Crisan-Dabija, ${ }^{1}$ Cristina Grigorescu, ${ }^{2}$ Cristina-Alice Pavel, ${ }^{3}$ Bogdan Artene, ${ }^{4}$ \\ Iolanda Valentina Popa $\mathbb{D}^{5,}{ }^{5,6}$ Andrei Cernomaz, ${ }^{7}$ and Alexandru Burlacu $\mathbb{D}^{4,6}$ \\ ${ }^{1}$ University of Medicine and Pharmacy “Grigore T. Popa” Iaşi, Pulmonology Department, Head of Clinic of Pulmonary Diseases, \\ Iasi, Romania \\ ${ }^{2}$ University of Medicine and Pharmacy "Grigore T. Popa” Iaşi, Department Thoracic Surgery, Clinic of Thoracic Surgery Iaşi, \\ Hospital of Pulmonary Diseases Iasi, Iasi, Romania \\ ${ }^{3}$ Clinic of Pulmonary Diseases, Iasi, Romania \\ ${ }^{4}$ Department of Interventional Cardiology-Cardiovascular Diseases Institute, Iasi, Romania \\ ${ }^{5}$ Institute of Gastroenterology and Hepatology, Iaşi, Romania \\ "Grigore T. Popa" University of Medicine, Iasi, Romania \\ ${ }^{7}$ University of Medicine and Pharmacy "Grigore T. Popa" Iaşi, Pulmonology Department, Institute of Oncology, Iaşi, Romania
}

Correspondence should be addressed to Iolanda Valentina Popa; iolivp@gmail.com

Received 5 April 2020; Revised 29 July 2020; Accepted 28 August 2020; Published 8 September 2020

Academic Editor: Jack Kastelik

Copyright (c) 2020 Radu Crisan-Dabija et al. This is an open access article distributed under the Creative Commons Attribution License, which permits unrestricted use, distribution, and reproduction in any medium, provided the original work is properly cited.

Background. The threat of contagious infectious diseases is constantly evolving as demographic explosion, travel globalization, and changes in human lifestyle increase the risk of spreading pathogens, leading to accelerated changes in disease landscape. Of particular interest is the aftermath of superimposing viral epidemics (especially SARS-CoV-2) over long-standing diseases, such as tuberculosis (TB), which remains a significant disease for public health worldwide and especially in emerging economies. Methods and Results. The PubMed electronic database was systematically searched for relevant articles linking TB, influenza, and SARS$\mathrm{CoV}$ viruses and subsequently assessed eligibility according to inclusion criteria. Using a data mining approach, we also queried the COVID-19 Open Research Dataset (CORD-19). We aimed to answer the following questions: What can be learned from other coronavirus outbreaks (focusing on TB patients)? Is coinfection (TB and SARS-CoV-2) more severe? Is there a vaccine for SARSCoV-2? How does the TB vaccine affect COVID-19? How does one diagnosis affect the other? Discussions. Few essential elements about TB and SARS-CoV coinfections were discussed. First, lessons from past outbreaks (other coronaviruses) and influenza pandemic/seasonal outbreaks have taught the importance of infection control to avoid the severe impact on TB patients. Second, although challenging due to data scarcity, investigating the pathological pathways linking TB and SARS-CoV-2 leads to the idea that their coexistence might yield a more severe clinical evolution. Finally, we addressed the issues of vaccination and diagnostic reliability in the context of coinfection. Conclusions. Because viral respiratory infections and TB impede the host's immune responses, it can be assumed that their lethal synergism may contribute to more severe clinical evolution. Despite the rapidly growing number of cases, the data needed to predict the impact of the COVID-19 pandemic on patients with latent TB and TB sequelae still lies ahead. The trial is registered with NCT04327206, NCT01829490, and NCT04121494.

\section{Introduction}

The global threat of contagious infectious diseases, particularly tuberculosis (TB), has long concerned authorities in charge of public health policies. Most data and all predictions concerning global epidemiology of TB are based on "real-life" analysis (surveys and national surveillance programs) conducted by the World Health Organization (WHO) [1, 2]. The incidence of TB is slowly declining but remains a significant issue worldwide (ranked as the ninth leading cause of death worldwide and the 


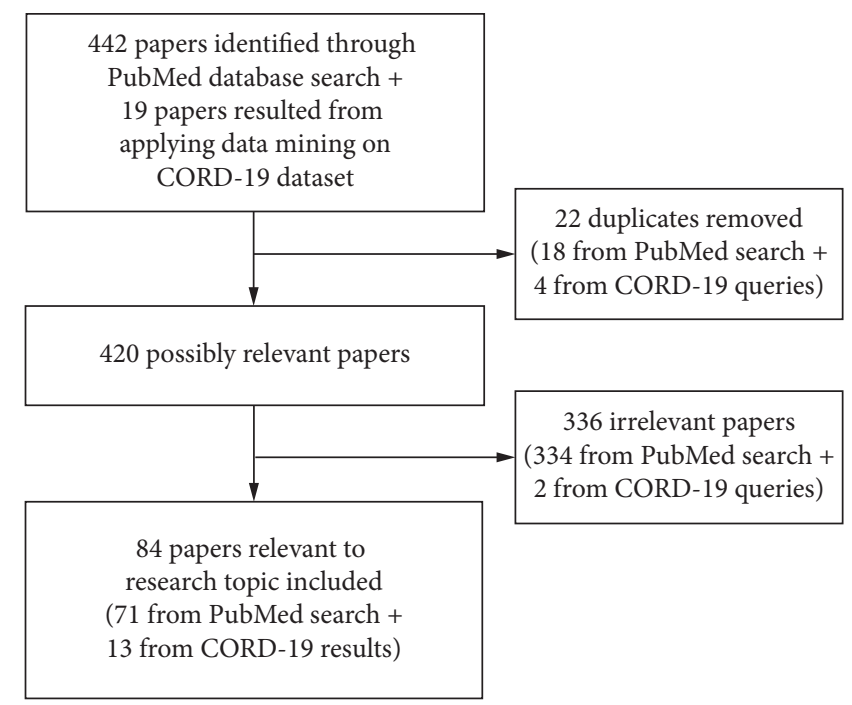

FIGURE 1: Study selection process and number of papers included.

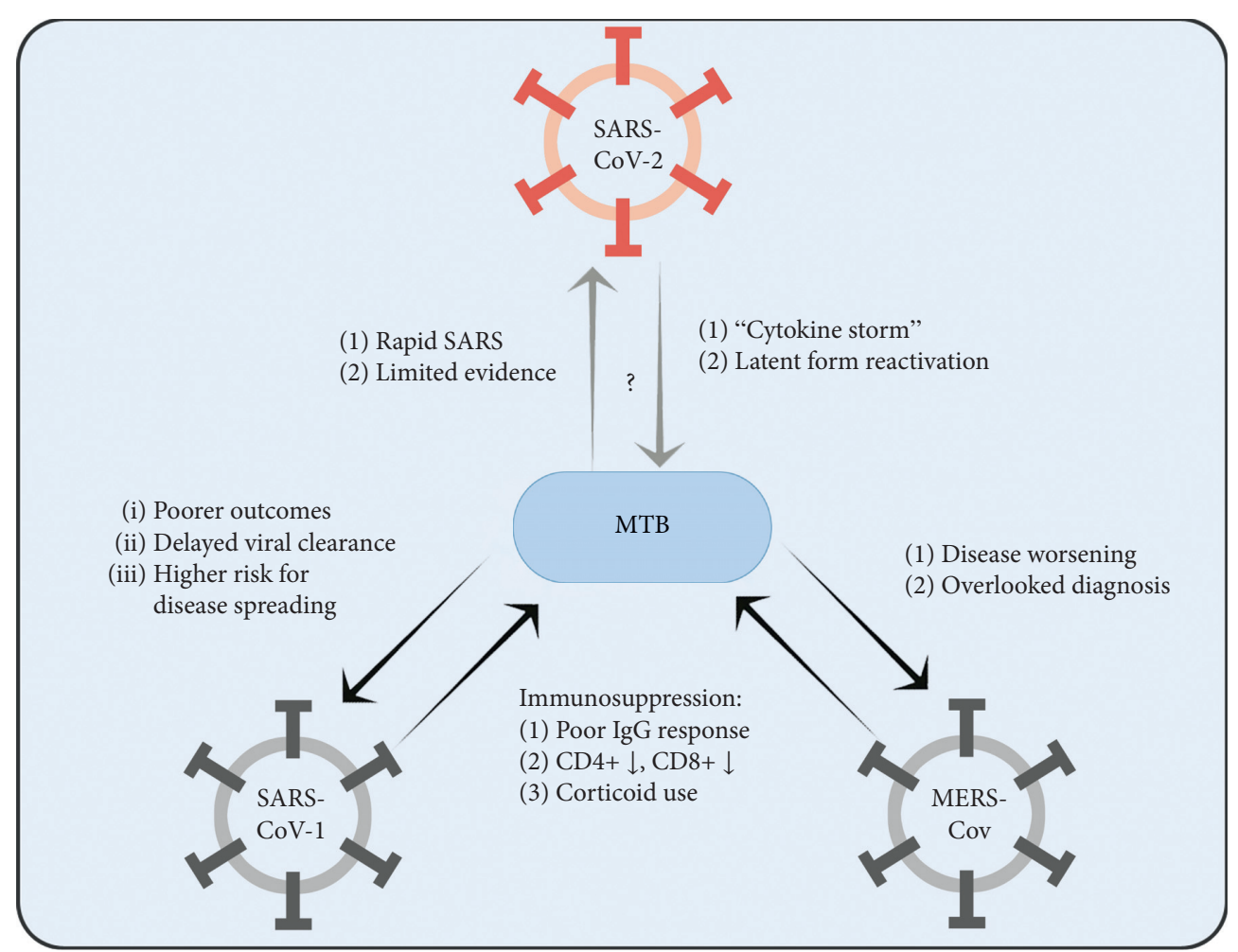

FIGURE 2: Known and possible interactions between MTB and coronaviruses.

leading cause of a single infectious agent $[3,4]$ ), especially in most middle-income and emerging-economy countries.

TB remains of great significance for the public health in Eastern Europe (e.g., Romania), which has the highest TB incidence in the European Union (EU) (4 times higher than the average), accounting for a quarter of the TB burden in the EU [4]. The incidence of TB increased in Romania after
1990, peaking in 2002 (142.2\%), with a downward trend since then, 54.5/100 000 in 2016, and 54.2\% lower than in $2002[4,5]$. A series of factors augmented the severity of TB endemic in Romania, namely, a large number of severe forms, cases with multidrug-resistant tuberculosis (MDRTB) and extensively drug-resistant TB (XDR-TB), HIV coinfection, and (TB-related) mortality in children. TB 
mortality in Romania has followed the same course as the incidence, with a peak in 2002 and an elevation of XDR-TB cases between 2012 and 2015, with a threefold increase [4].

Influenza infection may promote the progression of latent Mycobacterium tuberculosis (MTB) infection to active $\mathrm{TB}$, alter the clinical presentation of $\mathrm{TB}$, and also possibly exacerbate pulmonary TB (PTB) [6]. Both influenza and tuberculosis hinder host immune responses. Specifically, influenza can impair T-cell immunity and weaken innate immune responses against secondary bacterial infections $[6,7]$.

This deleterious synergism of viral and bacterial infections increases the risk of influenza-associated mortality, and patients with PTB may increase the severity of influenza disease and death due to chronic lung disease and immunosuppression. Epidemiologic data suggest an increased rate of influenza or severe influenza-associated disease in patients with TB during influenza pandemics $[6,8,9]$ or during seasonal influenza epidemics [10] compared with non-TB individuals.

\section{Objectives}

Individuals with chronic respiratory infections, including $\mathrm{TB}$, are first to experience the adverse effects of a pneumotropic pandemic, especially in the healthcare setting $[11,12]$. Given that both coronavirus disease 2019 (COVID19) and TB become important causes of mortality worldwide $[3,6,12]$ and the TB endemic situation in Romania [4], we sought to explore the possible outcomes of the inevitable collide of the two pandemics. Considering SARS-CoV-2 high transmissibility, it is very likely that COVID-19 will be of particular concern for individuals infected with MTB [13]. Also, coinfection with MTB is of particular importance as the TB diagnosis might be missed or shadowed by concern about COVID-19.

Therefore, we aimed to review the available literature in order to

(a) predict the impact of COVID-19 pandemic on patients with latent TB and TB sequelae based on the data available from the past influenza pandemic and seasonal influenza outbreaks (considering similar or more severe outcomes in the current pandemic)

(b) underline possible clinical particularities and diagnostic errors on these patients

(c) evaluate possible different therapeutic approaches on $\mathrm{TB}$ patients (latent, sequelae, or active) given that current COVID-19 treatment may induce mycobacterial proliferation [14]

\section{Methods}

The electronic database of PubMed was systematically searched for relevant articles from the inception until March 2020. The search terms used were ["tuberculosis" OR “TB”], AND ["flu” OR “influenza”], AND ["SARS” OR "SARS-CoV” OR "SARS-CoV-1"], AND MERS-CoV. The search process included article identification, removing the duplicates, screening titles and abstracts, and assessing eligibility of the selected full texts. Additionally, reference lists of valid articles were checked for studies of relevance. Articles were included if they involved data about past TB, SARS-CoV-1, MERS-CoV epidemics, TB-influenza viruses, and TB-SARS$\mathrm{CoV}-1$ coinfections or clinical or laboratory research on the immune responses during coinfections. Journal articles published with full text or abstracts in English were eligible for inclusion.

In order to identify emerging coinfection particularities of novel coronavirus SARS-CoV-1, we queried the COVID19 Open Research Dataset (CORD-19), the current largest open dataset available with over 47000 scholarly articles, including over 36000 with full text about COVID-19, SARSCoV-2, and other coronaviruses. The CORD-19 dataset is available at https://pages.semanticscholar.org/coronavirusresearch. Given the large quantity of textual data in CORD19, we applied a data mining approach to answer a few questions: (1) What can one learn from other coronaviruses epidemics (with a focus on TB patients)? (2) Is coinfection (TB and SARS-CoV-2) more severe? (3) Is there a vaccine for SARS-CoV-2? How does the TB vaccine influence COVID19? (4) How does one condition influence the diagnosis of the other one?

Articles were exported from CORD-19 and merged locally for further processing. Articles of interest were retrieved by administering the query "COVID" OR "COVID-19" OR "2019-nCoV" OR "SARS-CoV-2” OR "Novel coronavirus" OR "Tuberculosis" OR "Mycobacterium tuberculosis" OR "Flu” OR "Influenza” OR "Coinfection" OR "Vaccine" OR "Immunization." Data mining was further applied to select only articles that met our topics of interest about coinfections between particular pathogens stated earlier and COVID-19 developing vaccines. The study selection process and number of papers identified in each phase are illustrated in the flowchart (Figure 1).

\section{Discussions}

4.1. What Can One Learn from Other Past Epidemics/Pandemics? For a better understanding of managing a novel coronavirus pandemic, one needs to understand the experience. Since the first discovery of coronaviruses in 1960, there have been described three human coronaviruses known to cause fatal respiratory diseases:

(a) The severe acute respiratory syndrome (SARS) coronavirus (SARS-CoV, now known as SARS-CoV1) that led to a global epidemic in 2002 [13]

(b) The Middle-East respiratory syndrome coronavirus (MERS-CoV) which was discovered in 2012 and still affects people from 27 countries [15]

(c) Most recently, the novel coronavirus (SARS-CoV-2), whose outbreak led to an ongoing pandemic with thousands of new cases being confirmed each day and a growing number of reported deaths worldwide [13] (Figure 2)

It has to be added that while SARS-CoV-1 was associated in 37 countries with 8096 cases and 774 deaths 
during the entire nine months of the epidemic [13] and MERS with only 2494 cases and 858 deaths in 27 countries [13], SARS-CoV-2 spread (and still spreading) in 208 countries with 1009625 confirmed COVID-19 cases and 51737 confirmed deaths (as of the 3rd of April 2020) in only three months since the first declared case of COVID-19 pneumonia [16].

Its high transmissibility rate reminds of the 1918-19 influenza pandemic when it has been estimated that almost a third of the world's population is affected with a mortality rate of 2.5\% [9]. Other significant differences between influenza pandemics and current novel coronavirus pandemic can be found. One of the most notable is that death was less frequent amidst healthcare workers in influenza pandemics as it was the case in SARS, MERS, and now COVID-19 pandemic [17]. Despite this, other similarities still exist between SARS-CoV-2 and influenza as the striking resemblance of pathological features documented in COVID19 associated acute respiratory distress syndrome (ARDS) and H7N9-induced ARDS [18]. Also, it has been suggested that influenza viruses, as well as SARS-CoV-2, significantly upregulate angiotensin-converting enzyme 2 (ACE2) receptors. This upregulation facilitates novel coronavirus entrance into the host cell and makes patients infected by influenza more susceptible to SARS-CoV-2.

Some studies have shown that, in a patient with TB, induction of type I interferons (IFNs) determined by influenza infection could be detrimental [11], impeding the immune-competent host's ability to limit bacterial replication. Thus it promotes the infection [19] and precipitates TB mortality rate (pneumonia and influenza death rates among the age group most affected by TB exceeded in 1918 the TB mortality rate noted before and after the pandemic) [9]. Higher TB death rates were noted in winter months (coinciding with seasonal influenza outbreaks), which led to the suggestion of PTB being an independent risk factor for influenza-associated mortality [10]. In contrast, few studies suggested no association between influenza coinfection and PTB's progression or severity $[20,21]$. One murine model demonstrated that although influenza infection increased the IFN- $\gamma$ secretion, it had little effect on bacterial load in chronically infected mice with BCG [21]. Half of the analytical studies included in a recent systematic review showed no evidence on influenza affecting PTB presentation or its outcomes or, conversely, PTB affecting influenza presentation and outcomes [6]. Nevertheless, the magnitude of TB burden in a given setting might be an essential factor that should be considered in any study regarding PTB because its results might be biased [6].

During the 2002-03 SARS-CoV-1 epidemic, it was highlighted that to contain the epidemic, the correct management of symptomatic patients (within and outside the hospital) was critical [22]. The secondary transmission within Vancouver (Canada) was stopped due to the correct management of several imported cases, as opposed to other places (e.g., Toronto, Canada, or Taipei, Taiwan) where the incorrect management conducted to spread further and hospital clusters $[22,23]$. Also, inappropriate implementation of infection control strategies in Singapore led to massive healthcare personnel infection (half of the SARS cases were among healthcare workers) and several superspreading events [23].

TB in SARS patients has been reported in several studies from TB endemic countries such as Singapore, China, or Taiwan [24, 25], all with known TB patients that acquired SARS and in individuals that developed TB after recovery from SARS [25]. The transient immunosuppression characterized both conditions [26], a reason for poorer $\operatorname{IgG}$ antibody response and a delayed viral clearance in coinfected SARS patients [24]. Also, the use of corticoid therapy in SARS added even more on immunosuppression [24].

During an epidemic, many measures are taken (especially in hospitals) to limit the transmission of the disease to naïve patients. However, overcrowding hospitals are prone to mistakes. Known-TB patients from China supposedly acquired SARS due to exposure to SARS patients from the same hospital wards. Hence, coinfection could have been avoided [24]. Even though most of them recovered without complications, SARS coinfection on TB cases led to significantly lower mean CD4+ and CD8 + T cells and undetectable or unusually low antibody levels after SARS recovery [24]. Also, the viral excretion was two times longer in sputum and five times longer in stools for TB + SARS patients compared to SARS patients without TB, which translates into a higher potential to spread the virus [24].

When dealing with a possible SARS patient from an endemic TB region, one should never forget $\mathrm{TB}$ as a coexisting pathology. In April 2003, a SARS-related hospital screening from Taipei (Taiwan) resulted in discovering $60 \mathrm{~TB}$ cases among healthcare workers [27]. Moreover, during the SARS-CoV-1 epidemic from Singapore, SARS cases were reported developing active PTB short after recovering from SARS [25], data compatible with previous studies on mice regarding the suppression of cellular immunity after a viral infection [11]. There is also data on MERS-CoV augmenting TB by the added immunosuppression and reinforcing the need to evaluate a suspected patient [28].

\subsubsection{Key Points}

(1) Influenza pandemic/seasonal outbreaks and other coronaviruses epidemics might have a negative impact on TB patients.

(2) Transmission prevention was crucial for containing the epidemics.

(3) In order to decrease the opportunity of SARS-CoV-2 spreading among TB cases, hospital treatment for TB patients should be limited to severe cases.

4.2. Is Coinfection More Severe? Pathological Pathways Linking TB and SARS-CoV-2. Although the pathophysiology of SARS-CoV-2 is not fully understood, it seems most likely similar to the one of SARS-CoV-1. Substantial evidence suggests that SARS-CoV-2 infection could initiate an aggressive inflammation by increasing cytokines secretion such as interleukin- $1 \beta$ (IL- $1 \beta)$, interferon- $\gamma$ (IFN- $\gamma$ ), tumor 
necrosis factor- $\alpha$ (TNF- $\alpha$ ), interleukin-2 (IL-2), interleukin4 (IL-4), and interleukin-10 (IL-10), their plasma levels being associated with disease severity, leading to a so-called "cytokine storm," thus explaining some young adults' disease severity [29].

Immune system hyperreaction was also described in the 1918-1919 influenza pandemic, which was the first known pandemic to report an excess risk of death among individuals 25-35 years old [9]. Although cytokines storms seem to be induced by both SARS-CoV-2 and influenza, given the early stage in our understanding regarding SARS-CoV-2 infection, to conclude that the immunomodulatory or immune suppressive effects of these two viruses are highly similar might be premature, few studies proved that influenza aggravated the pulmonary status of individuals with TB so that latent TB could become active, a closed cavity might open, and various lesions might progress, leading to further deterioration of pulmonary function [9]. In this regard, a mouse coronavirus model demonstrated the ability to reactivate dormant MTB from CD271 + mesenchymal stem cells through the altruistic stem cell-based defense mechanism, predicting a potential increase of TB in SARS-CoV-2 era. Additionally, in a cohort of 49 patients with active TB and COVID-19, the diagnosis of COVID-19 preceded or was simultaneous (within seven days) with TB in 23 patients, raising the suspicion that SARS-CoV-2 infection might boost the development of active TB. However, this remains purely speculative as individuals with latent TB infection were not followed up over time [30].

Cytokines have an essential role in host resistance to TB infection, being first demonstrated in murine infection models [19] and later validated by severe mycobacterial disease findings in patients with mutations in the IFN- $\gamma$ and IL-12 signaling pathways and rheumatoid arthritis or Crohn's disease patients treated with TNF- $\alpha$ blockade $[19,31]$.

Since the SARS-CoV-2 is a newly discovered pathogen (first infection being reported in December 2019) [29], little data about the coinfection with MTB could be found (especially considering the long incubation period of MTB from exposure to developing the disease, often with a slow onset) $[32,33]$. Still, the existent studies showed that TB status might play a role in the development of severe acute respiratory syndrome in SARS-CoV-2 coinfection, considering the cases described in China and India [34]. A recent meta-analysis [35] concluded that patients with TB are not more likely to get COVID-19, but TB is associated with a 2.1fold increased risk of severe COVID-19 disease, although the statistical difference was not significant. Moreover, no increased risk for mortality in coincident COVID-19 and TB was found. However, this study included a small number of TB patients infected with SARS-CoV-2, and the publication bias was not rigorously assessed. Thus, the findings should be interpreted with caution. Similarly, in a cohort of 69 patients, in all cases, COVID-19 contributed to worsen the prognosis of TB patients and/or cause death, although TB was not a significant determinant of mortality [36].

On the contrary, 20 patients with TB and COVID-19 had a rather benign clinical course of the coinfection, with only one patient that died. TB lesions at chest X-ray were not aggravated, and only four patients had signs of newly developed pneumonia [37].

One should keep in mind that the existence of underlying conditions, autoimmune diseases, poor hygiene, and overcrowding is all known as risk factors for developing one, another, or both diseases $[29,32]$. In a paper developing a model of pathogen dissemination in the outpatient clinic, it was suggested that populations with a high risk of contracting influenza or SARS might also have a higher prevalence of MTB [38]. It is no coincidence that the regions with the highest TB burden, as reported by the World Health Organization, were predicted to be hardest hit by the social and economic consequences of COVID-19 [39].

Another serious problem posed by the COVID-19 pandemic is the treatment continuity of TB patients. The nature of the disease, with extended treatment regimens and poor outcomes with drug resistance resulting from therapy discontinuation, are significant problems even in regular times, all the more in a pandemic context with numerous and stringent isolation measures [40]. This shifts the directly observed therapy to self-administered therapy, for which digital-health technologies such as electronic medication monitors and video-supported therapy were recommended to ensure adherence to treatments [41]. Discontinuation risks and other challenges faced by the tuberculosis clinical trials in the face of COVID-19 have been discussed, sounding the alarm around these threats [42].

\subsubsection{Key Points}

(1) Cytokines seem to play an essential role in both COVID-19 and TB, their plasma level being associated with disease's severity.

(2) Immune system hyperreaction could explain a more unfortunate outcome in people 25-35 years old.

(3) Although there is limited data on MTB and COVID19 coinfection, one could reasonably presume that their coexistence might have a more severe evolution for the patient.

4.3. (Proven or Presumed) Clinical and Paraclinical Impacts of Vaccination. One of the most effective ways to prevent diseases caused by pathogens, like bacteria or viruses, proved to be vaccination [43]. Since the first discovery of SARS, extensive research was done to find a vaccine to prevent the disease [44]. Different vaccine types were tested: inactivated or live-attenuated virus, DNA-based vaccines, recombinant proteins, virus-like particles, and viral vectors with some promising efficiency, but with neither being finally approved for use $[44,45]$. Recent data suggest that the SARS-CoV-2 genome is up to $80 \%$ similar to SARS-CoV- 1 and up to $50 \%$ similar to MERS-CoV [46], so previous studies on protective immune responses SARS-CoV-1 or MERS-CoV may aid vaccine development for SARS-CoV-2 [47]. Considering that there is no approved vaccine neither for SARS-CoV-1 nor for MERS-CoV, other options are considered, such as the vaccine used for TB prevention [48]. 
Since 1921 a vaccine is used widely for TB prevention, a live-attenuated strain of the bovine tubercle bacillus named bacillus Calmette-Guerin (BCG) [49, 50]. In 1927, it was observed that BCG-vaccinated newborns had a three times lower mortality rate in their first year of life than the unvaccinated ones [51]. Later was noted a decrease in infectious morbidities, protecting both mice (against secondary fungal or parasitic infections with Candida albicans or Schistosoma mansoni through tissue macrophages activation) [49] and infants (against acute lower respiratory infections). Thus, the risk of acute lower respiratory infections in BCG-vaccinated infants seemed to be $37 \%$ lower than in unvaccinated controls among children $<5$ years old $[49,52]$. However, there seem to be no proven data regarding the duration of these beneficial effects [53], and there is data suggesting that subsequent administration of different vaccines was associated with altering the nonspecific immunity [54], so one might safely presume that the chance of a BCG vaccine received decades ago in childhood could influence the course of one pandemic in adulthood which would be low.

Although one might argue that the lack of widespread BCG vaccination in the United States may be influencing the course of their pandemic compared to countries with broad spread vaccination, one should also keep in mind that the United States delayed the implementation of infection control strategies (that could avoid superspreading events). There still is a reluctance of face masks wearing when out in public, a measure that has been proven to slow and stop the spread of the virus [55].

There is data suggesting that BCG vaccination of adults could increase the capacity of producing proinflammatory cytokines such as Il- $1 \beta$ and IL- 6 , which leads to nonspecific protection against unrelated pathogens like Staphylococcus aureus or Candida albicans [56].

Considering these facts, the BCG vaccine is contemplated as a potential candidate against respiratory viruses [48]. Moreover, Muldron Children's Research Institute from Australia already announced a phase III randomized controlled trial, which will determine if healthcare workers' BCG vaccination will have any impact on SARS-CoV-2 infection (BCG Vaccination to Protect Healthcare Workers against COVID-19, BRACE, NCT04327206). However, more time is needed to establish its supposed efficiency.

Given the high TB burden, especially in emerging economies and the high global threat of SARS-CoV-2, a vaccine that may be beneficial in combating TB and COVID19 would be of high interest.

Adenoviral vectors have previously been used to improve immunogenicity with excellent results in the enhancement of both humoral and cellular immunity [57]. ChAdOx1 85A has been contemplated as a TB vaccine first in healthy vaccinated BCG adults (Phase I Trial to Evaluate the Safety and Immunogenicity of a ChAdOx1 85A Vaccination with and without MVA85A Boost in Healthy BCG Vaccinated Adults, NCT01829490) and most recently with an ongoing trial in healthy adults with or without prior BCG vaccination (A Phase I Clinical Trial to Compare the Safety and Immunogenicity of Candidate TB Vaccine ChAdOx1 85A Administered by the Aerosol Inhaled Route and the
Intramuscular Route in Healthy Adult Subjects, NCT04121494).

The University of Oxford appears to be repurposing this viral-based TB vaccine for use against SARS-CoV-2 by changing the immunogenetic antigen expressed. ChAdOx1 nCoV-19 (more recently known as AZD1222) is a replication-deficient simian adenoviral vector expressing the fulllength SARS-CoV-2 spike (S) protein. In rhesus macaques, ChAdOx1 nCoV-19 induced both humoral and cellular immune responses after one single dose. In humans, the preliminary results demonstrated an acceptable safety profile and spike-specific T cell responses as early as day 7, peaking on day 14 , and maintained up to day 56 [57]. The neutralizing antibody responses were observed in up to $91 \%$ of the cases after one single dose and up to $100 \%$ after a booster dose [19]. 10560 healthy UK volunteers are expected to be enrolled in a phase II/III clinical trial that already begun and they will undergo follow-up for one-year after enrollment (A Phase 2/3 Study to Determine the Efficacy, Safety, and Immunogenicity of the Candidate Coronavirus Disease (COVID-19) Vaccine ChAdOx1 nCoV-19, NCT04327206).

It is noted that SARS-CoV-2 envelope spike (S) protein has a decisive role for determining host tropism and transmission capacity [46] and T cell epitopes-based peptide derived from $S$ proteins that map to SARS-CoV-2 proteins [47] and subunit vaccines based on $S$ protein are also considered for preventing SARS-CoV-2 infection [47, 58, 59].

Novel methods are emerging such as reverse vaccinology that refers to the process of constructing vaccines by detecting viral antigens through genomic analysis using bioinformatics tools. Reverse vaccinology has successfully been applied to fight against the Zika virus or Chikungunya virus. One study proposed reverse vaccinology and immunoinformatics methods to design potential subunit vaccines against SARS-CoV-2 using the highly antigenic viral proteins and epitopes. Suggested vaccine constructs appeared to confer good immunogenic response through various computational studies. Three vaccine constructs were designed, and the best one was selected through molecular docking study. Another study proposes a specific synthetic vaccine epitope and peptidomimetic agent, identified through bioinformatics methods [60].

Currently, there are 15 potential vaccine candidates for SARS-CoV-2 in the pipeline globally developed using various technologies (messenger RNA, synthetic DNA, synthetic, and modified virus-like particles) [61, 62].

\subsubsection{Key Points}

(1) SARS-CoV-2 genome is up to $80 \%$ similar to SARS$\mathrm{CoV}-1$ and $50 \%$ similar to MERS-CoV.

(2) No SARS vaccine was approved for clinical use (in 18 years of research).

(3) Ongoing trials on the SARS-CoV-2 vaccine are on the highest interest.

4.4. Diagnostic Errors in the Context of COVID-19 and TB Coexistence (or How Does One Condition Influence the 
Diagnosis of the Other?). TB and COVID-19 are mainly respiratory diseases that primarily affect the lungs; however, the onset of TB is often slow compared to COVID-19, which seems to develop in a few days from exposure [4, 22]. Given the clinical and imagistic similarities such as cough, fever, or shortness of breath and various radiological pulmonary lesions $[4,22]$, accurate diagnostic tests should be made available to avoid overlooking one condition in favor of the other one.

Tuberculin skin test (TST) and with a greater sensibility and specificity, the interferon-gamma release assays (IGRAs) are widely used for TB screening [63]. Given their results are influenced by the host's immune response after MTB (or BCG) exposure [64], there is a gap for diagnostic errors in individuals with an impaired immune system, such as in a concurrent severe infection [65, 66]. Increased age, low peripheral lymphocyte count, high body mass index, and immunosuppressive therapies were also associated with falsenegative results [66] that could lead to missing TB diagnose. Moreover, an excess of inflammatory markers could affect IGRAs sensitivity, and the high value of C-reactive protein (CRP) might be a confounder for false-negative results [67].

It has been observed that high CRP and low peripheral lymphocyte counts could occur within a few days of exposure to SARS-CoV-2 [68]. Therefore, this observation may lead to the possibility that a patient with latent TB or TB sequel may have a false-negative IGRA.

As SARS-CoV-2 has not been identified for a few months in humans, there is no specific treatment [13]. Given the growing number of reported cases, suspected patients must be diagnosed as quickly as possible to isolate and limit further transmission [13]. Conventional methods such as assays for detecting viral antigens or antiviral antibodies and newer methods of diagnosis as multiplex nucleic acid amplification have been developed and used clinically [13].

With the urge of identifying the radiological features of SARS-CoV-2 infection, with the community transmission present in most countries, and with its nonspecific clinical onset (fever, dry cough, dyspnoea and radiological findings of bilateral infiltrates, and even pleural effusion and cavitation) [69], doctors may either be facing a steep differential diagnostic or not consider tuberculosis at all.

Considering the sudden onset of the SARS-CoV-2 pandemic, countries struggled to quickly find a possible treatment to prevent respiratory failures and deaths, especially among patients with respiratory comorbidities. Also, since its fatal dynamics, there is no time to carry out new drug development in the traditional manner. Therefore, screening for already available drugs (for any activity against SARS-CoV-2) [13] is usually preferred in the first instance. It seems that an antiviral used for HIV infection, composed of two protease inhibitors (lopinavir and ritonavir), would have a therapeutic effect on coronavirus infections. It seems to have entered as a recommendation in the treatment of the COVID-19 in a short time [13]. Other compounds, such as redexivir, favivir, ribavirin, nitrazine, and chloroquine/ hydroxychloroquine, are evaluated [13, 68]. Chloroquine and hydroxychloroquine have been shown to shorten the duration of SARS-CoV-2 viremia by reducing the viral load [68]. However, hydroxychloroquine has also been associated with a higher risk of nontuberculous mycobacterial (NTM) infection in rheumatoid arthritis patients [14].

\subsubsection{Key Points}

(1) Coinfection of TB and SARS-CoV-2 may be challenging to diagnose.

(2) SARS-CoV-2 infection may mask the clinical and radiological active $\mathrm{TB}$.

(3) Patients receiving the proposed treatment for COVID-19 may be at risk for the infection with NTM.

\section{Conclusions}

Because viral respiratory infections and $\mathrm{TB}$ impede the host's immune responses, their lethal synergism can be assumed to contribute to more severe clinical evolution. Coinfection most likely affects both sides of these patients: rapid development of severe acute respiratory syndrome through cytokine-mediated immune response and increased risk of tuberculosis reactivation. As a lesson from previous outbreaks, hospital treatment for patients with tuberculosis should be limited to severe cases, to prevent the spread of SARS-CoV-2 in TB cases. Despite the rapidly increasing number of cases, the data needed to predict the impact of the COVID-19 pandemic on patients with latent TB and TB sequelae and to guide management in this particular context still lies ahead.

\section{Data Availability}

Data used to support the findings of this study are available from the corresponding author upon request.

\section{Disclosure}

Cristina Grigorescu has the same contribution as the first author.

\section{Conflicts of Interest}

The authors declare that there are no conflicts of interest regarding the publication of this article.

\section{Acknowledgments}

This study was funded by the Romanian Academy of MedAcademy of Medical Sciences and European Regional Development Fund (MySMIS 107124: Funding Contract 2/ Axa 1/31.07.2017/107124 SMIS).

\section{References}

[1] M. Zignol, W. van Gemert, D. Falzon et al., "Surveillance of anti-tuberculosis drug resistance in the world: an updated analysis, 2007-2010," Bulletin of the World Health Organization, vol. 90, no. 2, pp. 111-119, 2012. 
[2] R. J. Coker, "Review: multidrug-resistant tuberculosis: public health challenges," Tropical Medicine and International Health, vol. 9, no. 1, pp. 25-40, 2004.

[3] J. Beauté, M. Dara, P. Colombani, S. Ehsani, O. Gozalov, and A. Hovanesyan, Tuberculosis Surveillance and Monitoring in Europe 2017, Vol. 150, European Centre for Disease Prevention and Control, Stockholm, Sweden, 2017.

[4] A.-L. Golli, M. F. Niţu, F. Turcu, M. Popescu, L. CiobanuMitrache, and M. Olteanu, "Tuberculosis remains a public health problem in Romania," The International Journal of Tuberculosis and Lung Disease, vol. 23, no. 2, pp. 226-231, 2019.

[5] F. M. NiŢu, M. Olteanu, C. T Streba et al., "Tuberculosis and its particularities in Romania and worldwide," Romanian journal of morphology and embryology=Revue roumaine de morphologie et embryologie, vol. 58, no. 2, pp. 385-392, 2017.

[6] S. Walaza, C. Cohen, S. Tempia et al., "Influenza and tuberculosis co-infection: a systematic review," Influenza and Other Respiratory Viruses, vol. 14, no. 1, pp. 77-91, 2020.

[7] C.-L. Small, C. R. Shaler, S. McCormick et al., "Influenza infection leads to increased susceptibility to subsequent bacterial superinfection by impairing NK cell responses in the lung," The Journal of Immunology, vol. 184, no. 4, pp. 2048-2056, 2010.

[8] A. Noymer, "The 1918 influenza pandemic hastened the decline of tuberculosis in the United States: an age, period, cohort analysis," Vaccine, vol. 29, no. 2, pp. B38-B41, 2011.

[9] W. Oei and H. Nishiura, "The relationship between tuberculosis and influenza death during the influenza (H1N1) pandemic from 1918-19," Computational and Mathematical Methods in Medicine, vol. 2012, Article ID 124861, 2012.

[10] S. Walaza, C. Cohen, A. Nanoo, A. L. Cohen, J. McAnerney, and C. von Mollendorf, "Excess mortality associated with influenza among tuberculosis deaths in South Africa, 1999-2009," PLoS One, vol. 10, no. 6, Article ID e0129173, 2015.

[11] P. S. Redford, K. D. Mayer-Barber, F. W. McNab et al., "Influenza A virus impairs control of Mycobacterium tuberculosis coinfection through a type I interferon receptor-dependent pathway," The Journal of Infectious Diseases, vol. 209, no. 2, pp. 270-274, 2014.

[12] W. H. Organisation, WHO Information on Tuberculosis and Pandemic Influenza A (H1N1), WHO, Geneva, Switzerland, 2009, http://www9.who.int/tb/features_archive/h1n1/en/.

[13] J.-Y. Li, Z. You, Q. Wang et al., "The epidemic of 2019-novelcoronavirus (2019-nCoV) pneumonia and insights for emerging infectious diseases in the future," Microbes and Infection, vol. 22, no. 2, pp. 80-85, 2020.

[14] S. K. Brode, F. B. Jamieson, R. Ng et al., "Increased risk of mycobacterial infections associated with anti-rheumatic medications," Thorax, vol. 70, no. 7, pp. 677-682, 2015.

[15] W. H. Organization, "MERS situation update," 2020, http://www.emro.who.int/pandemic-epidemic-diseases/ mers-cov/mers-situation-update-january-2020.html.

[16] W. H. Organization, "Coronavirus disease (COVID-19) pandemic," 2019, https://www.who.int/emergencies/diseases/ novel-coronavirus-2019.

[17] J. Rello, S. Tejada, C. Userovici, K. Arvaniti, J. Pugin, and G. Waterer, "Coronavirus disease 2019 (COVID-19): a critical care perspective beyond China," Anaesthia, Critical Care \& Pain Medicine, vol. 39, no. 3, pp. 167-169, 2020.

[18] J. Chen, C. Hu, L. Chen, L. Tang, Y. Zhu, and X. Xu, Clinical Study of Mesenchymal Stem Cell Treating Acute Respiratory Distress Syndrome Induced by Epidemic Influenza A (H7N9)
Infection, a Hint for COVID-19 Treatment, Engineering, Beijing, China, 2020.

[19] K. D. Mayer-Barber and A. Sher, "Cytokine and lipid mediator networks in tuberculosis," Immunological Reviews, vol. 264, no. 1, pp. 264-275, 2015.

[20] S. Roth, S. Whitehead, S. Thamthitiwat et al., "Concurrent influenza virus infection and tuberculosis in patients hospitalized with respiratory illness in Thailand," Influenza and Other Respiratory Viruses, vol. 7, no. 3, pp. 244-248, 2013.

[21] D. O. Co, L. H. Hogan, J. Karman et al., "Interactions between T cells responding to concurrent mycobacterial and influenza infections," The Journal of Immunology, vol. 177, no. 12, pp. 8456-8465, 2006.

[22] D. Raoult, A. Zumla, F. Locatelli, G. Ippolito, and G. Kroemer, "Coronavirus infections: epidemiological, clinical and immunological features and hypotheses," Cell Stress, vol. 4, no. 4, pp. $66-75,2020$.

[23] D. S. C. Hui and A. Zumla, "Severe acute respiratory syndrome," Infectious Disease Clinics of North America, vol. 33, no. 4, pp. 869-889, 2019.

[24] L. Wei, F. Arnaud, Z. Pan-He, Z. Lin, X. Zhong-Tao, and T. Fang, "Pulmonary tuberculosis and SARS, China," Emerging Infectious Disease Journal, vol. 12, no. 4, p. 707, 2006.

[25] J. G. H. Low, C. C. Lee, Y. S. Leo, J. Guek-Hong Low, C.-C. Lee, and Y.-S. Leo, "Severe acute respiratory syndrome and pulmonary tuberculosis," Clinical Infectious Diseases, vol. 38, no. 12, pp. e123-e125, 2004.

[26] T. Li, Z. Qiu, L. Zhang et al., "Significant changes of peripheral T lymphocyte subsets in patients with severe acute respiratory syndrome," The Journal of Infectious Diseases, vol. 189, no. 4, pp. 648-651, 2004.

[27] "Nosocomial transmission of Mycobacterium tuberculosis found through screening for severe acute respiratory syndrome-Taipei, Taiwan, 2003," MMWR Morbidity and Mortality Weekly Report, vol. 53, no. 15, pp. 321-322, 2004.

[28] S. H. Alfaraj, J. A. Al-Tawfiq, T. A. Altuwaijri, and Z. A. Memish, "Middle East respiratory syndrome coronavirus and pulmonary tuberculosis coinfection: implications for infection control," Intervirology, vol. 60, no. 1-2, pp. 53-55, 2017.

[29] W. J. Guan, Z. Y. Ni, Y. Hu, W. H. Liang, C. Q. Ou, and J. X. He, "Clinical characteristics of coronavirus disease 2019 in China," The New England Journal of Medicine, vol. 382, pp. 1708-1720, 2020.

[30] M. Tadolini, L. R. Codecasa, J.-M. García-García et al., "Active tuberculosis, sequelae and COVID-19 co-infection: first cohort of 49 cases," European Respiratory Journal, vol. 56, no. 1, Article ID 2001398, 2020.

[31] J. Harris and J. Keane, "How tumour necrosis factor blockers interfere with tuberculosis immunity," Clinical and Experimental Immunology, vol. 161, no. 1, pp. 1-9, 2010.

[32] W. H. Organization, Addressing the Needs of Vulnerable Populations, 2020, https://www.who.int/tb/areas-of-work/ population-groups/en/.

[33] K. Dheda, T. Gumbo, N. R. Gandhi et al., "Global control of tuberculosis: from extensively drug-resistant to untreatable tuberculosis," The Lancet Respiratory Medicine, vol. 2, no. 4, pp. 321-338, 2014.

[34] S. Yasri and V. Wiwanitkit, "Tuberculosis and novel Wuhan coronavirus infection: pathological interrelationship," Indian Journal of Tuberculosis, vol. 67, no. 2, p. 264, 2020.

[35] Y. Gao, M. Liu, Y. Chen, S. Shi, J. Geng, and J. Tian, “Association between tuberculosis and COVID-19 severity and 
mortality: a rapid systematic review and meta-analysis," Journal of Medical Virology, 2020.

[36] I. Motta, R. Centis, L. D’Ambrosio et al., “Tuberculosis, COVID-19 and migrants: preliminary analysis of deaths occurring in 69 patients from two cohorts," Pulmonology, vol. 26, no. 4, pp. 233-240, 2020.

[37] C. Stochino, S. Villa, P. Zucchi, P. Parravicini, A. Gori, and M. C. Raviglione, "Clinical characteristics of COVID-19 and active tuberculosis co-infection in an Italian reference hospital," European Respiratory Journal, vol. 56, no. 1, Article ID 2001708, 2020.

[38] R. H. John, G. S. David, and S. C. Philip, "Pathogen transmission and clinic scheduling," Emerging Infectious Disease Journal, vol. 12, no. 1, p. 159, 2006.

[39] E. Harding, "WHO global progress report on tuberculosis elimination," The Lancet Respiratory Medicine, vol. 8, no. 1, p. 19, 2020.

[40] F. Amimo, B. Lambert, and A. Magit, "What does the COVID-19 pandemic mean for HIV, tuberculosis, and malaria control?" Tropical Medicine and Health, vol. 48, no. 1, p. 32, 2020.

[41] T. Manyazewal, Y. Woldeamanuel, H. M. Blumberg, A. Fekadu, and V. C. Marconi, "The fight to end tuberculosis must not be forgotten in the COVID-19 outbreak," Nature Medicine, vol. 26, no. 6, pp. 811-812, 2020.

[42] I. D. Rusen, "Challenges in tuberculosis clinical trials in the face of the COVID-19 pandemic: a sponsor's perspective," Tropical Medicine and Infectious Disease, vol. 5, no. 2, p. 86, 2020.

[43] W. H. Organisation, Coronavirus Disease (COVID-19) Pandemic, 2019, https://www.who.int/topics/vaccines/en/.

[44] R. L. Graham, E. F. Donaldson, and R. S. Baric, "A decade after SARS: strategies for controlling emerging coronaviruses," Nature Reviews Microbiology, vol. 11, no. 12, pp. 836-848, 2013.

[45] N. Zhang, S. Jiang, and L. Du, "Current advancements and potential strategies in the development of MERS-CoV vaccines," Expert Review of Vaccines, vol. 13, no. 6, pp. 761-774, 2014.

[46] R. Lu, X. Zhao, J. Li et al., "Genomic characterisation and epidemiology of 2019 novel coronavirus: implications for virus origins and receptor binding," The Lancet, vol. 395, no. 10224, pp. 565-574, 2020.

[47] S. F. Ahmed, A. A. Quadeer, and M. R. McKay, "Preliminary identification of potential vaccine targets for the COVID-19 coronavirus (SARS-CoV-2) based on SARS-CoV immunological studies," Viruses, vol. 12, no. 3, 2020.

[48] J. A. Soto, N. M. S. Gálvez, C. A. Rivera, C. E. Palavecino, P. F. Céspedes, and E. Rey-Jurado, "Recombinant BCG vaccines reduce pneumovirus-caused airway pathology by inducing protective humoral immunity," Frontiers in Immunology, vol. 9, no. 2875, 2018.

[49] A. Angelidou, J. Diray-Arce, M. G. Conti, K. K. Smolen, S. D. van Haren, and D. J. Dowling, "BCG as a case study for precision vaccine development: lessons from vaccine heterogeneity, trained immunity, and immune ontogeny," Frontiers in Microbiology, vol. 11, no. 332, 2020.

[50] P. Andersen and T. M. Doherty, "The success and failure of BCG - implications for a novel tuberculosis vaccine," Nature Reviews Microbiology, vol. 3, no. 8, pp. 656-662, 2005.

[51] P. Aaby and C. S. Benn, "Saving lives by training innate immunity with bacille Calmette-Guerin vaccine," Proceedings of the National Academy of Sciences, vol. 109, no. 43, pp. 17317-17318, 2012.
[52] M.-G. Hollm-Delgado, E. A. Stuart, and R. E. Black, “Acute lower respiratory infection among Bacille Calmette-Guérin (BCG)-vaccinated children," Pediatrics, vol. 133, no. 1, pp. e73-e81, 2014.

[53] A. J. Pollard, A. Finn, and N. Curtis, "Non-specific effects of vaccines: plausible and potentially important, but implications uncertain," Archives of Disease in Childhood, vol. 102, no. 11, pp. 1077-1081, 2017.

[54] N. Curtis, A. Sparrow, T. A. Ghebreyesus, and M. G. Netea, "Considering BCG vaccination to reduce the impact of COVID-19," The Lancet, vol. 395, no. 10236, pp. 1545-1546, 2020.

[55] D. K. Chu, E. A. Akl, S. Duda et al., "Physical distancing, face masks, and eye protection to prevent person-to-person transmission of SARS-CoV-2 and COVID-19: a systematic review and meta-analysis," The Lancet, vol. 395, no. 10242, pp. 1973-1987, 2020.

[56] C. Covián, A. Fernández-Fierro, A. Retamal-Díaz, F. E. Díaz, A. E. Vasquez, and M. K. Lay, "BCG-induced cross-protection and development of trained immunity: implication for vaccine design," Frontiers in Immunology, vol. 10, no. 2806, 2019.

[57] P. M. Folegatti, K. J. Ewer, P. K. Aley, B. Angus, S. Becker, and S. Belij-Rammerstorfer, "Safety and immunogenicity of the ChAdOx1 nCoV-19 vaccine against SARS-CoV-2: a preliminary report of a phase $1 / 2$, single-blind, randomised controlled trial," The Lancet, vol. 369, no. 10249, pp. 467-478, 2020.

[58] N. Wang, J. Shang, S. Jiang, and L. Du, "Subunit vaccines against emerging pathogenic human coronaviruses," Frontiers in Microbiology, vol. 11, no. 298, 2020.

[59] S. Kuate, J. Cinatl, H. W. Doerr, and K. Überla, "Exosomal vaccines containing the $S$ protein of the SARS coronavirus induce high levels of neutralizing antibodies," Virology, vol. 362, no. 1, pp. 26-37, 2007.

[60] B. Robson, "Computers and viral diseases. Preliminary bioinformatics studies on the design of a synthetic vaccine and a preventative peptidomimetic antagonist against the SARSCoV-2 (2019-nCoV, COVID-19) coronavirus," Computers in Biology and Medicine, vol. 119, Article ID 103670, 2020.

[61] J. Pang, M. X. Wang, I. Y. H. Ang et al., "Potential rapid diagnostics, vaccine and therapeutics for 2019 novel coronavirus (2019-ncoV): a systematic review," Journal of Clinical Medicine, vol. 9, no. 3, p. 623, 2020.

[62] K. K. Eriksson, D. Makia, R. Maier, B. Ludewig, and V. Thiel, "Towards a coronavirus-based HIV multigene vaccine," Clinical \& Developmental Immunology, vol. 13, no. 2-4, pp. 353-360, 2006.

[63] L. Di and Y. Li, "The risk factor of false-negative and falsepositive for T-SPOT.TB in active tuberculosis," Journal of Clinical Laboratory Analysis, vol. 32, no. 2, Article ID e22273, 2018.

[64] L. L. Lu, M. T. Smith, K. K. Q. Yu et al., "IFN- $\gamma$-independent immune markers of Mycobacterium tuberculosis exposure," Nature Medicine, vol. 25, no. 6, pp. 977-987, 2019.

[65] E. Whittaker, E. López-Varela, C. Broderick, and J. A. Seddon, "Examining the complex relationship between tuberculosis and other infectious diseases in children," Frontiers in Pediatrics, vol. 7, no. 233, 2019.

[66] M. Yamasue, K. Komiya, Y. Usagawa, K. Umeki, S.-i. Nureki, and M. Ando, "Factors associated with false negative interferon- $\gamma$ release assay results in patients with tuberculosis: a systematic review with meta-analysis," Scientific Reports, vol. 10, no. 1, p. 1607, 2020. 
[67] Y.-S. Kwon, Y. H. Kim, K. Jeon et al., "Factors that predict negative results of quantiferon-tb gold in-tube test in patients with culture-confirmed tuberculosis: a multicenter retrospective cohort study," PLoS One, vol. 10, no. 6, Article ID e0129792, 2015.

[68] W. Zhang, Y. Zhao, F. Zhang et al., "The use of anti-inflammatory drugs in the treatment of people with severe coronavirus disease 2019 (COVID-19): the Perspectives of clinical immunologists from China," Clinical Immunology, vol. 214, Article ID 108393, 2020.

[69] S. Salehi, A. Abedi, S. Balakrishnan, and A. Gholamrezanezhad, "Coronavirus disease 2019 (COVID19): a systematic review of imaging findings in 919 patients," American Journal of Roentgenology, vol. 215, no. 1, pp. 1-7, 2020 . 